\title{
A New Corresponding-States Model for the Correlation and Prediction of the Surface Tension of Organic Acids
}

\author{
Isidro Cachadiña* and Ángel Mulero* \\ Departamento de Física Aplicada, Universidad de Extremadura, 06006 Badajoz, Spain \\ E-mail: icacha@unex.es; mulero@unex.es
}

Supporting Information Available 
Table S1: Data set used in the determination of coefficients $a_{1}, n_{1}$ and $n_{2}$ in Eq. (19). When no surface tension is available at the triple point temperature, Eq. (19) is used with $T_{t}=T_{\min }$ and $\sigma_{t}=\sigma\left(T_{\min }\right)$. In these cases the value of $\sigma_{t}$ taken is underlined. ${ }^{A}$ Strong acidity and ${ }^{a}$ weak acidity classification. ${ }^{15}$

\begin{tabular}{|c|c|c|c|c|c|c|c|c|}
\hline Fluid & Name & CAS & $N$ & $T_{c}(\mathrm{~K})$ & $T_{t}(\mathrm{~K})$ & $T_{\min }(\mathrm{K})$ & $T_{\max }(\mathrm{K})$ & $\sigma_{t}(\mathrm{mN} / \mathrm{m})$ \\
\hline 1 & n-pentadecanoic acid ${ }^{a}$ & $1002-84-2$ & 16 & 774 & 325.68 & 325.68 & 697.5 & 29.8997 \\
\hline 2 & n-undecanoic acid & $112-37-8$ & 13 & 732 & 301.63 & 301.63 & 658.8 & 30.7291 \\
\hline 3 & n-octanoic acid ${ }^{a}$ & $124-07-2$ & 32 & 694.26 & 289.65 & 289.65 & 624.83 & 29.4525 \\
\hline 4 & n-hexanoic acid ${ }^{a}$ & $142-62-1$ & 40 & 660.2 & 269.25 & 270.15 & 593.19 & $\underline{30.1083}$ \\
\hline 5 & n-dodecanoic acid ${ }^{a}$ & $143-07-7$ & 38 & 743 & 316.98 & 316.98 & 668.7 & 28.8432 \\
\hline 6 & n-decanoic acid ${ }^{a}$ & $334-48-5$ & 28 & 722.1 & 304.55 & 304.55 & 649.89 & 29.603 \\
\hline 7 & n-heptadecanoic acid ${ }^{a}$ & $506-12-7$ & 20 & 792 & 334.25 & 334.25 & 675.75 & 29.3162 \\
\hline 8 & n-eicosanic acid ${ }^{a}$ & $506-30-9$ & 11 & 820 & 348.23 & 348.4 & 738 & 29.29 \\
\hline 9 & n-tetradecanoic acid ${ }^{a}$ & $544-63-8$ & 24 & 763 & 327.37 & 327.4 & 422.45 & 28.61 \\
\hline 10 & n-hexadecanoic acid ${ }^{a}$ & $57-10-3$ & 26 & 785 & 335.66 & 335.9 & 423.15 & $\underline{28.78}$ \\
\hline 11 & n-octadecanoic acid ${ }^{a}$ & $57-11-4$ & 29 & 803 & 342.75 & 342.75 & 685.51 & 28.3732 \\
\hline 12 & n-tridecanoic acid & $638-53-9$ & 14 & 754 & 315.01 & 315.01 & 678.6 & 29.8072 \\
\hline 13 & $\operatorname{acetic}_{\text {acid }^{a}}$ & 64-19-7 & 165 & 591.95 & 289.81 & 290.1 & 583.15 & $\underline{28.1}$ \\
\hline 14 & n-nonadecanoic acid & $646-30-0$ & 20 & 811 & 341.23 & 341.23 & 729.9 & $\overline{29.4646}$ \\
\hline 15 & benzoic acid ${ }^{A}$ & $65-85-0$ & 22 & 751 & 395.45 & 395.45 & 675.45 & 31.93 \\
\hline 16 & propionic acid & $79-09-4$ & 87 & 600.81 & 252.45 & 252.75 & 573.15 & 30.95 \\
\hline 17 & acrylic acid & $79-10-7$ & 23 & 615 & 286.15 & 286.15 & 546.15 & 29.62 \\
\hline
\end{tabular}


Table S2: Fluids with no available surface tension data at temperatures closer than $1 \mathrm{~K}$ from the triple point temperature, except for formic acid. The value of $\sigma_{\min }$ used, with $T_{\min }$ in Eq. (24), is given. ${ }^{A}$ Strong acidity and ${ }^{a}$ weak acidity classification. ${ }^{15}$

\begin{tabular}{|c|c|c|c|c|c|c|c|c|}
\hline Fluid & Name & CAS & $N$ & $T_{c}(\mathrm{~K})$ & $T_{t}(\mathrm{~K})$ & $T_{\min }(\mathrm{K})$ & $T_{\max }(\mathrm{K})$ & $\sigma_{\min }(\mathrm{mN} / \mathrm{m})$ \\
\hline 18 & n-butyric acid ${ }^{a}$ & $107-92-6$ & 97 & 615.7 & 267.95 & 273.15 & 523.15 & 27.75 \\
\hline 19 & n-pentanoic acid ${ }^{a}$ & $109-52-4$ & 36 & 639.16 & 239.15 & 253.15 & 503.15 & 30.79 \\
\hline 20 & n-heptanoic acid ${ }^{a}$ & $111-14-8$ & 30 & 677.3 & 265.83 & 273.15 & 573.15 & 29.84 \\
\hline 21 & n-nonanoic acid ${ }^{a}$ & $112-05-0$ & 16 & 710.7 & 285.55 & 293.15 & 639.63 & 29.7 \\
\hline 22 & oleic acid ${ }^{a}$ & $112-80-1$ & 17 & 781 & 286.53 & 293.15 & 453.15 & 32.5 \\
\hline 23 & o-toluic acid ${ }^{A}$ & $118-90-1$ & 7 & 751 & 376.85 & 413.15 & 473.15 & 31.7 \\
\hline 24 & levulinic acid ${ }^{A}$ & $123-76-2$ & 11 & 738 & 308.15 & 313.15 & 388.15 & 38.64 \\
\hline 25 & $\begin{array}{l}\text { cyclopropane } \\
\text { boxylic acid }{ }^{A}\end{array}$ & $1759-53-1$ & 4 & 671 & 290.65 & 295.55 & 359.05 & 34.29 \\
\hline 26 & isovaleric acid $^{A}$ & $503-74-2$ & 35 & 629.09 & 243.85 & 273.15 & 573.15 & 27.34 \\
\hline 27 & formic acid ${ }^{A}$ & 64-18-6 & 74 & 588 & 281.45 & 282.35 & 483.15 & 38.1 \\
\hline 28 & isobutyric acid $^{a}$ & $79-31-2$ & 46 & 605 & 227.15 & 253.15 & 503.15 & 28.83 \\
\hline \multirow[t]{2}{*}{29} & m-toluic acid ${ }^{A}$ & $99-04-7$ & 6 & 754 & 384.15 & 413.15 & 473.15 & 31.7 \\
\hline & Number of total data & & 379 & & & & & \\
\hline
\end{tabular}


Table S3: Data of the fluids with more than $90 \%$ of the data being predicted using the Sugden ${ }^{46}$ method.

\begin{tabular}{|c|c|c|c|c|c|c|c|c|}
\hline Fluid & Name & CAS & $N$ & $T_{c}(\mathrm{~K})$ & $T_{t}(\mathrm{~K})$ & $T_{\min }(\mathrm{K})$ & $T_{\max }(\mathrm{K})$ & $\sigma_{\min }(\mathrm{mN} / \mathrm{m})$ \\
\hline 30 & terephthalic acid & $100-21-0$ & 15 & 883.6 & 700.15 & 700.15 & 795.24 & 26.67 \\
\hline 31 & 4-methoxyphenylacetic acid & $104-01-8$ & 11 & 768 & 356.15 & 356.15 & 691.2 & 24.317 \\
\hline 32 & trans-crotonic acid & $107-93-7$ & 11 & 666 & 344.55 & 344.55 & 594.55 & 26.128 \\
\hline 33 & succinic acid & $110-15-6$ & 39 & 838 & 460.85 & 460.85 & 828.33 & 29.761 \\
\hline 34 & maleic acid & $110-16-7$ & 11 & 773 & 403.8 & 403.8 & 695.7 & 36.651 \\
\hline 35 & fumaric acid & $110-17-8$ & 10 & 771 & 562.55 & 573.15 & 690.15 & 16.77 \\
\hline 36 & glutaric acid & $110-94-1$ & 50 & 840 & 371.65 & 371.65 & 827.99 & 36.3745 \\
\hline 37 & diglycolic acid & $110-99-6$ & 11 & 820 & 421.15 & 421.15 & 731.15 & 52.26 \\
\hline 38 & pimelic acid & $111-16-0$ & 20 & 842 & 378.15 & 378.15 & 830.11 & 32.5923 \\
\hline 39 & sebacic acid & $111-20-6$ & 39 & 845 & 406.25 & 406.25 & 833.75 & 27.2289 \\
\hline 40 & cyclopentylacetic acid & $1123-00-8$ & 11 & 708 & 286.65 & 286.65 & 637.2 & 35.533 \\
\hline 41 & 2,6-naphthalenedicarboxylic acid & $1141-38-4$ & 11 & 904 & 640.15 & 640.15 & 813.6 & 17.182 \\
\hline 42 & 2-methylbutyric acid & $116-53-0$ & 11 & 643 & 193 & 193 & 573 & 38.62 \\
\hline 43 & neoheptanoic acid & $1185-39-3$ & 11 & 662 & 230 & 230 & 595.8 & 34.696 \\
\hline 44 & 6-hydroxyhexanoic acid & $1191-25-9$ & 11 & 730 & 313.15 & 313.15 & 657 & 25.841 \\
\hline
\end{tabular}


Table S3: continued from previous page

\begin{tabular}{|c|c|c|c|c|c|c|c|c|}
\hline Fluid & Name & CAS & $N$ & $T_{c}(\mathrm{~K})$ & $T_{t}(\mathrm{~K})$ & $T_{\min }(\mathrm{K})$ & $T_{\max }(\mathrm{K})$ & $\sigma_{\min }(\mathrm{mN} / \mathrm{m})$ \\
\hline 45 & 2-formyl benzoic acid & $119-67-5$ & 11 & 772 & 370.15 & 370.15 & 694.8 & 75.633 \\
\hline 46 & isophthalic acid & $121-91-5$ & 11 & 1007 & 619.15 & 619.15 & 899.15 & 49.08 \\
\hline 47 & azelaic acid & $123-99-9$ & 39 & 844 & 381.25 & 381.25 & 832.13 & 30.2749 \\
\hline 48 & adipic acid & $124-04-9$ & 16 & 841 & 426.55 & 426.55 & 756.9 & 28.655 \\
\hline 49 & pyruvic acid & $127-17-3$ & 11 & 634.52 & 286.75 & 286.75 & 566.75 & 47.56 \\
\hline 50 & pimaric acid & $127-27-5$ & 20 & 807.1 & 491.65 & 491.65 & 726.39 & 25.602 \\
\hline 51 & tartaric acid & $133-37-9$ & 11 & 828 & 479.15 & 479.15 & 739.15 & 54.78 \\
\hline 52 & acetoxyacetic acid & $13831-30-6$ & 15 & 764 & 341.15 & 363.8 & 687.89 & 54.2 \\
\hline 53 & cinnamic acid & $140-10-3$ & 38 & 797 & 406.15 & 406.15 & 776.96 & 32.641 \\
\hline 54 & malonic acid & $141-82-2$ & 39 & 834 & 409.15 & 409.15 & 823.11 & 21.6133 \\
\hline 55 & oxalic acid & $144-62-7$ & 39 & 828 & 462.65 & 462.65 & 818.63 & 11.6524 \\
\hline 56 & 2-ethyl hexanoic acid & $149-57-5$ & 16 & 674.6 & 155.15 & 235 & 607.14 & 33.155 \\
\hline 57 & ibuprofen & $15687-27-1$ & 11 & 765 & 348.62 & 348.62 & 688.5 & 29.668 \\
\hline 58 & monomethyl terephthalate & $1679-64-7$ & 16 & 797.8 & 492.54 & 492.54 & 718.02 & 36.17 \\
\hline 59 & dehydroabietic acid & $1740-19-8$ & 11 & 828 & 445.65 & 445.65 & 745.2 & 26.215 \\
\hline 60 & dilactic acid & 19201-34-4 & 11 & 660 & 385.65 & 385.65 & 594 & 18.852 \\
\hline
\end{tabular}


Table S3: continued from previous page

\begin{tabular}{|c|c|c|c|c|c|c|c|c|}
\hline Fluid & Name & CAS & $N$ & $T_{c}(\mathrm{~K})$ & $T_{t}(\mathrm{~K})$ & $T_{\min }(\mathrm{K})$ & $T_{\max }(\mathrm{K})$ & $\sigma_{\min }(\mathrm{mN} / \mathrm{m})$ \\
\hline 61 & palustric acid & $1945-53-5$ & 19 & 789 & 441.65 & 441.65 & 770.72 & 21.655 \\
\hline 62 & acryloxy propionic acid & $24615-84-7$ & 15 & 729.5 & 283.8 & 283.8 & 656.55 & 42.122 \\
\hline 63 & 2-methyloctanoic acid & $3004-93-1$ & 14 & 694 & 240 & 240 & 661.57 & 34.892 \\
\hline 64 & 4-hydroxymethyl benzoic acid & $3006-96-0$ & 12 & 814.4 & 454.65 & 454.65 & 732.96 & 32.649 \\
\hline 65 & 2-methylhexanoic acid & $4536-23-6$ & 15 & 666 & 230 & 230 & 599.4 & 35.253 \\
\hline 66 & linolenic acid & $463-40-1$ & 11 & 780 & 262.05 & 262.05 & 692.05 & 36.18 \\
\hline 67 & neoabietic acid & $471-77-2$ & 11 & 812 & 446.4 & 446.4 & 730.8 & 25.738 \\
\hline 68 & citraconic acid & $498-23-7$ & 11 & 829 & 356.15 & 356.15 & 736.15 & 56.53 \\
\hline 69 & lactic acid & $50-21-5$ & 11 & 675 & 289.9 & 291.15 & 561.15 & 42.599 \\
\hline 70 & cis-crotonic acid & $503-64-0$ & 6 & 647 & 288.65 & 288.65 & 433.65 & 27.46 \\
\hline 71 & suberic acid & $505-48-6$ & 20 & 843 & 416.45 & 416.45 & 832.06 & 28.6688 \\
\hline 72 & acetylsalicylic acid & $50-78-2$ & 11 & 765 & 408.15 & 408.15 & 688.5 & 38.032 \\
\hline 73 & ascorbic acid & $50-81-7$ & 11 & 783 & 465.15 & 465.15 & 695.15 & 41.04 \\
\hline 74 & abietic acid & $514-10-3$ & 11 & 832 & 446.65 & 446.65 & 646.65 & 28.738 \\
\hline 75 & trimellitic acid & $528-44-9$ & 10 & 860 & 511.15 & 511.15 & 747.71 & 15.011 \\
\hline 76 & acetoacetic acid & $541-50-4$ & 15 & 678.5 & 310.65 & 310.65 & 610.65 & 36.884 \\
\hline
\end{tabular}


Table S3: continued from previous page

\begin{tabular}{|c|c|c|c|c|c|c|c|c|}
\hline Fluid & Name & CAS & $N$ & $T_{c}(\mathrm{~K})$ & $T_{t}(\mathrm{~K})$ & $T_{\min }(\mathrm{K})$ & $T_{\max }(\mathrm{K})$ & $\sigma_{\min }(\mathrm{mN} / \mathrm{m})$ \\
\hline 77 & isopimaric acid & $5835-26-7$ & 20 & 807.1 & 435.65 & 435.65 & 726.39 & 31.446 \\
\hline 78 & alpha-hydroxyisobutyric acid & $594-61-6$ & 11 & 664 & 352.15 & 352.15 & 597.6 & 22.828 \\
\hline 79 & neohexanoic acid & $595-37-9$ & 11 & 653 & 259.15 & 259.15 & 587.7 & 30.324 \\
\hline 80 & linoleic acid & $60-33-3$ & 11 & 775 & 268.15 & 268.15 & 688.15 & 34.996 \\
\hline 81 & hydroxycaproic acid & $6064-63-7$ & 11 & 683 & 334 & 334 & 614.7 & 29.854 \\
\hline 82 & 1,4-cyclohexanedicarboxylic acid & $619-82-9$ & 16 & 819.8 & 583 & 583 & 737.82 & 17.318 \\
\hline 83 & methoxyacetic acid & $625-45-6$ & 11 & 691 & 281 & 281 & 621 & 41.82 \\
\hline 84 & malic acid & $6915-15-7$ & 11 & 781 & 403.15 & 403.15 & 693.15 & 26.66 \\
\hline 85 & dodecanedioic acid & $693-23-2$ & 16 & 847 & 401.15 & 401.15 & 762.3 & 28.24 \\
\hline 86 & salicylic acid & $69-72-7$ & 11 & 739 & 431.75 & 431.75 & 661.75 & 30.73 \\
\hline 87 & $\begin{array}{l}\text { octahydro-pentalene-1-carboxylic } \\
\text { acid }\end{array}$ & $7403-22-7$ & 16 & 799.5 & 312.9 & 312.9 & 719.55 & 50.532 \\
\hline 88 & neopentanoic acid & $75-98-9$ & 10 & 632 & 309.08 & 309.08 & 568.8 & 22.795 \\
\hline 89 & citric acid & $77-92-9$ & 15 & 800.2 & 427.1 & 427.1 & 720.18 & 11.239 \\
\hline 90 & trilactic acid & $78024-33-6$ & 10 & 777 & 312.15 & 312.15 & 660.58 & 40.212 \\
\hline 91 & glycolic acid & $79-14-1$ & 11 & 616 & 352.65 & 352.65 & 552.65 & 36.82 \\
\hline
\end{tabular}


Table S3: continued from previous page

\begin{tabular}{llrllllll} 
Fluid & Name & $\mathrm{CAS}$ & $N$ & $T_{c}(\mathrm{~K})$ & $T_{t}(\mathrm{~K})$ & $T_{\min }(\mathrm{K})$ & $T_{\max }(\mathrm{K})$ & $\sigma_{\min }(\mathrm{mN} / \mathrm{m})$ \\
\hline 92 & peracetic acid & $79-21-0$ & 11 & 552 & 272.95 & 272.95 & 492.95 & 39.8 \\
93 & methacrylic acid & $79-41-4$ & 11 & 662 & 288.15 & 288.15 & 595.8 & 29.904 \\
94 & tetradecanedioic acid & $821-38-5$ & 16 & 849 & 399.15 & 399.15 & 764.1 & 28.249 \\
95 & 2-ethyl butyric acid & $88-09-5$ & 11 & 655 & 258.15 & 258.15 & 588.15 & 50.43 \\
96 & phthalic acid & $88-99-3$ & 11 & 800 & 464.15 & 464.15 & 714.15 & 26.85 \\
97 & pyromellitic acid & $89-05-4$ & 11 & 893 & 554 & 554 & 794 & 25.35 \\
98 & itaconic acid & $97-65-4$ & 11 & 821 & 438.75 & 438.75 & 738.75 & 41.15 \\
99 & p-toluic acid & $99-94-5$ & 16 & 775 & 452.34 & 452.34 & 697.14 & 33.749 \\
\hline & Number of total data & \multicolumn{7}{c}{1079} \\
\hline
\end{tabular}


Table S4: Absolute average deviations (AAD\%) of the correlation considered in this work for the fluids with more than $90 \%$ of data predicted. Sastri and Rao (SR) Eq. (2), ${ }^{21}$ Miqueu et al (MQ) Eq. (3), ${ }^{22}$ Di Nicola and Moglie (DM1,DM2) Eqs. (4) and (6), ${ }^{23}$ Gharagheizi al (GH) Eq. (7), ${ }^{24}$ Di Nicola et al (DCP1,DCP2) Eq. (8), ${ }^{15}$ and this work Eq. (24). AADs greater than 10\% are written in boldface. The minimum AAD correlation value of each fluid is underlined. Terephthalic acid has no prediction for models SR and GH because it has no normal boiling point.

\begin{tabular}{|c|c|c|c|c|c|c|c|c|c|c|}
\hline & Name & $N$ & $\mathrm{SR}$ & MIQ & DM1 & DM2 & $\mathrm{GH}$ & DCP1 & DCP2 & Eq. (24) \\
\hline 30 & terephthalic acid & 15 & - & 30.27 & 54.47 & 47.87 & - & 43.33 & 25.07 & 4.00 \\
\hline 32 & trans-crotonic acid & 11 & $\underline{13.45}$ & 90.36 & 51.00 & 60.64 & 42.82 & 33.73 & 77.00 & 20.09 \\
\hline 33 & succinic acid & 39 & 10.62 & 60.05 & 24.49 & 36.38 & 20.05 & 7.69 & 34.72 & $\underline{7.28}$ \\
\hline 35 & fumaric acid & 10 & 19.70 & 56.00 & 5.80 & 25.10 & 2.00 & 10.30 & 46.60 & $\underline{0.40}$ \\
\hline 36 & glutaric acid & 50 & 14.92 & 49.62 & 18.28 & 28.24 & 13.96 & 13.18 & 16.62 & $\underline{3.36}$ \\
\hline 37 & diglycolic acid & 11 & 40.36 & 20.73 & 22.36 & 7.18 & 26.27 & 18.36 & 10.91 & $\underline{6.73}$ \\
\hline 40 & cyclopentylacetic acid & 11 & 14.45 & 28.82 & 11.36 & 17.64 & 8.82 & 8.45 & 19.00 & $\underline{4.00}$ \\
\hline 41 & $\begin{array}{l}\text { 2,6-naphthalenedicarboxylic } \\
\text { acid }\end{array}$ & 11 & 16.36 & 42.00 & 3.00 & 17.36 & 2.18 & 7.27 & 36.36 & $\underline{1.64}$ \\
\hline 42 & 2-methylbutyric acid & 11 & $\underline{3.27}$ & 47.64 & 28.18 & 35.55 & 22.55 & 6.82 & 25.09 & 6.45 \\
\hline
\end{tabular}




\begin{tabular}{|c|c|c|c|c|c|c|c|c|c|c|}
\hline & & & & & & & Table $s$ & : continu & from pre & vious page \\
\hline & Name & $N$ & $\mathrm{SR}$ & MIQ & DM1 & DM2 & $\mathrm{GH}$ & DCP1 & DCP2 & Eq. (24) \\
\hline 43 & neoheptanoic acid & 11 & 8.55 & 33.09 & 15.55 & 21.82 & 14.09 & 10.55 & 18.09 & $\underline{6.18}$ \\
\hline 44 & 6-hydroxyhexanoic acid & 11 & 25.45 & 116.27 & 54.82 & 88.00 & 51.18 & 33.91 & 65.91 & $\underline{1.18}$ \\
\hline 45 & 2-formyl benzoic acid & 11 & 60.91 & 30.27 & 49.36 & 43.55 & 51.18 & 47.82 & 34.64 & $\underline{7.36}$ \\
\hline 46 & isophthalic acid & 11 & 51.09 & $\underline{1.36}$ & 37.73 & 26.73 & 39.27 & 25.73 & 11.45 & 6.18 \\
\hline 47 & azelaic acid & 39 & 14.77 & 42.74 & 13.00 & 21.23 & 12.10 & 13.90 & 15.00 & $\underline{4.33}$ \\
\hline 48 & adipic acid & 16 & $\underline{3.38}$ & 63.56 & 25.00 & 37.62 & 22.81 & 7.00 & 29.25 & 6.75 \\
\hline 49 & pyruvic acid & 11 & 38.45 & 8.64 & 14.09 & 7.09 & 20.09 & 34.27 & 13.91 & $\underline{5.36}$ \\
\hline 50 & pimaric acid & 20 & 44.90 & 31.30 & 41.30 & 37.85 & 32.95 & 47.35 & 32.80 & $\underline{5.05}$ \\
\hline 51 & tartaric acid & 11 & 41.73 & 39.09 & 30.18 & 5.09 & 33.64 & 35.45 & 18.09 & $\underline{1.27}$ \\
\hline 52 & acetoxyacetic acid & 15 & 44.53 & $\underline{4.33}$ & 25.93 & 19.13 & 29.93 & 27.53 & 9.93 & 6.20 \\
\hline 53 & cinnamic acid & 38 & 27.05 & 19.34 & 7.34 & 6.63 & 7.79 & 3.66 & 32.00 & $\underline{1.45}$ \\
\hline 54 & malonic acid & 39 & 49.10 & 168.23 & 106.44 & 130.46 & 95.95 & 53.56 & 113.72 & $\underline{3.69}$ \\
\hline 55 & oxalic acid & 39 & 134.49 & 244.85 & 218.54 & 229.13 & 211.23 & 150.15 & 257.31 & $\underline{3.74}$ \\
\hline 56 & 2-ethyl hexanoic acid & 16 & 9.50 & 30.50 & 12.50 & 21.56 & 11.25 & 5.81 & 15.19 & $\underline{2.19}$ \\
\hline 57 & ibuprofen & 11 & $\underline{3.27}$ & 39.64 & 23.00 & 41.82 & 28.55 & 7.91 & 23.73 & 8.64 \\
\hline 58 & monomethyl terephthalate & 16 & 48.25 & 12.56 & 36.62 & 29.00 & 36.81 & 31.63 & 14.06 & $\underline{3.12}$ \\
\hline 59 & dehydroabietic acid & 11 & 26.36 & 4.09 & 21.00 & 13.55 & 11.09 & 25.36 & 10.09 & $\underline{0.36}$ \\
\hline
\end{tabular}




\begin{tabular}{|c|c|c|c|c|c|c|c|c|c|c|}
\hline & & & & & & & Tables & 4: continu & from pre & vious page \\
\hline & Name & $N$ & SR & MIQ & DM1 & DM2 & GH & DCP1 & DCP2 & Eq. (24) \\
\hline 60 & dilactic acid & 11 & $\underline{3.64}$ & 53.00 & 25.36 & 42.45 & 24.09 & 10.00 & 40.45 & 4.09 \\
\hline 61 & palustric acid & 19 & 28.95 & 15.42 & 25.00 & 17.74 & 14.95 & 27.42 & $\underline{8.63}$ & 13.00 \\
\hline 62 & acryloxy propionic acid & 15 & 20.33 & 30.80 & 2.40 & 12.47 & $\underline{1.47}$ & 7.27 & 19.00 & 6.67 \\
\hline 63 & 2-methyloctanoic acid & 14 & 12.43 & 26.07 & 9.57 & 16.29 & 9.21 & 5.00 & 26.00 & $\underline{3.79}$ \\
\hline 64 & 4-hydroxymethyl benzoic acid & 12 & 21.42 & 41.25 & 6.92 & 25.42 & 4.92 & 14.42 & 8.67 & $\underline{4.83}$ \\
\hline 65 & 2-methylhexanoic acid & 15 & 8.87 & 34.27 & 15.13 & 23.60 & 13.47 & 4.53 & 23.93 & 3.67 \\
\hline 66 & linolenic acid & 11 & 10.64 & 16.64 & 12.73 & 2.91 & $\underline{2.55}$ & 9.00 & 19.18 & 7.09 \\
\hline 67 & neoabietic acid & 11 & 29.45 & 10.45 & 24.91 & 19.18 & 15.00 & 30.82 & 14.18 & $\underline{0.55}$ \\
\hline 68 & citraconic acid & 11 & 34.27 & 29.00 & 13.64 & 1.73 & 17.91 & 27.73 & 13.73 & 10.18 \\
\hline 69 & lactic acid & 11 & 18.73 & 54.00 & 13.91 & 36.18 & 7.55 & 22.18 & $\underline{2.36}$ & 5.82 \\
\hline 70 & cis-crotonic acid & 6 & 8.33 & 82.17 & 49.50 & 58.83 & 38.17 & 12.67 & 41.17 & 2.50 \\
\hline 71 & suberic acid & 20 & 16.65 & 42.50 & 13.65 & 21.65 & 12.30 & 14.45 & 16.30 & $\underline{4.45}$ \\
\hline 72 & acetylsalicylic acid & 11 & 38.73 & 1.55 & 23.45 & 14.91 & 23.91 & 25.64 & 10.45 & 2.00 \\
\hline 73 & ascorbic acid & 11 & 26.55 & 63.27 & 16.09 & 32.00 & 18.91 & 21.91 & $\underline{4.73}$ & 7.45 \\
\hline 74 & abietic acid & 11 & 32.09 & 4.36 & 25.55 & 15.91 & 18.55 & 31.27 & 18.36 & $\underline{0.82}$ \\
\hline 75 & trimellitic acid & 10 & 80.10 & 236.00 & 111.30 & 173.60 & 110.40 & 116.50 & 164.60 & 11.30 \\
\hline 76 & acetoacetic acid & 15 & 17.60 & 42.13 & 11.60 & 22.73 & 5.33 & 7.87 & 21.13 & $\underline{4.33}$ \\
\hline
\end{tabular}




\begin{tabular}{|c|c|c|c|c|c|c|c|c|c|c|}
\hline & & & & & & & Table $s$ & 4: continu & from pre & vious page \\
\hline & Name & $N$ & SR & MIQ & DM1 & DM2 & $\mathrm{GH}$ & DCP1 & DCP2 & Eq. (24) \\
\hline 77 & isopimaric acid & 20 & 44.25 & 30.35 & 40.35 & 36.90 & 32.20 & 41.05 & 27.60 & $\underline{3.60}$ \\
\hline 78 & alpha-hydroxyisobutyric acid & 11 & 12.55 & 98.82 & 50.27 & 77.45 & 43.00 & 6.09 & 41.09 & $\underline{2.91}$ \\
\hline 79 & neohexanoic acid & 11 & 15.64 & 26.09 & 13.27 & 16.00 & 9.82 & 16.82 & $\underline{8.00}$ & 8.55 \\
\hline 80 & linoleic acid & 11 & 18.09 & 14.27 & 20.00 & 7.82 & 12.27 & 14.45 & 22.73 & $\underline{2.91}$ \\
\hline 81 & hydroxycaproic acid & 11 & 9.00 & 56.27 & 12.91 & 36.09 & 9.73 & 2.18 & 25.91 & $\underline{1.45}$ \\
\hline 82 & 1,4-cyclohexanedicarboxylic & 16 & 18.81 & 37.25 & 3.31 & 18.12 & 2.38 & $\underline{1.69}$ & 29.56 & 2.25 \\
\hline & acid & & & & & & & & & \\
\hline 83 & methoxyacetic acid & 11 & 20.82 & 44.09 & 9.45 & 17.36 & 3.27 & 8.18 & 19.73 & $\underline{0.91}$ \\
\hline 84 & malic acid & 11 & 21.18 & 147.91 & 54.27 & 105.73 & 47.55 & 26.36 & 60.18 & $\underline{2.64}$ \\
\hline 85 & dodecanedioic acid & 16 & 13.12 & 36.25 & 5.50 & 11.81 & 4.81 & 20.94 & 5.88 & $\underline{2.31}$ \\
\hline 86 & salicylic acid & 11 & 26.18 & 24.64 & 5.18 & 14.36 & 4.27 & 10.45 & 14.64 & $\underline{3.82}$ \\
\hline 87 & $\begin{array}{l}\text { octahydro-pentalene-1- } \\
\text { carboxylic acid }\end{array}$ & 16 & 33.81 & $\underline{5.38}$ & 15.25 & 9.38 & 16.37 & 26.06 & 13.25 & 7.75 \\
\hline 88 & neopentanoic acid & 10 & 9.30 & 63.20 & 42.60 & 48.10 & 38.40 & 7.70 & 40.30 & $\underline{6.00}$ \\
\hline 89 & citric acid & 15 & 173.00 & 403.07 & 222.67 & 376.13 & 219.87 & 123.87 & 181.27 & $\underline{14.00}$ \\
\hline 90 & trilactic acid & 10 & 14.00 & 42.10 & 5.10 & 19.30 & 4.00 & 11.10 & 9.00 & $\underline{3.00}$ \\
\hline 91 & glycolic acid & 11 & 27.00 & 52.91 & 5.45 & 27.36 & 4.00 & 18.18 & 9.55 & $\underline{1.45}$ \\
\hline
\end{tabular}




\begin{tabular}{|c|c|c|c|c|c|c|c|c|c|c|}
\hline & \multirow[b]{2}{*}{ Name } & \multirow[b]{2}{*}{$N$} & \multirow[b]{2}{*}{ SR } & \multirow[b]{2}{*}{ MIQ } & \multirow[b]{2}{*}{ DM1 } & \multirow[b]{2}{*}{ DM2 } & \multicolumn{4}{|c|}{ Table S4: continued from previous page } \\
\hline & & & & & & & GH & DCP1 & DCP2 & Eq. (24) \\
\hline 92 & peracetic acid & 11 & 28.55 & 24.64 & 4.36 & 14.91 & 6.45 & 27.64 & 8.45 & $\underline{0.55}$ \\
\hline 93 & methacrylic acid & 11 & $\underline{3.45}$ & 49.36 & 34.27 & 36.27 & 30.36 & 16.45 & 53.64 & 7.91 \\
\hline 94 & tetradecanedioic acid & 16 & 12.87 & 32.88 & 8.06 & 9.94 & 4.75 & 25.75 & 6.31 & $\underline{3.44}$ \\
\hline 95 & 2-ethyl butyric acid & 11 & 38.82 & $\underline{6.64}$ & 21.27 & 16.64 & 23.55 & 34.55 & 18.45 & 8.09 \\
\hline 96 & phthalic acid & 11 & 15.27 & 48.73 & 8.18 & 27.73 & 5.82 & 1.91 & 26.64 & $\underline{1.45}$ \\
\hline 97 & pyromellitic acid & 11 & 9.64 & 68.55 & 5.09 & 35.09 & 4.00 & $\underline{0.64}$ & 22.64 & 3.36 \\
\hline 98 & itaconic acid & 11 & 32.18 & 31.82 & 11.36 & $\underline{1.91}$ & 15.45 & 12.91 & 14.82 & 6.09 \\
\hline 99 & p-toluic acid & 16 & 38.44 & $\underline{0.69}$ & 20.19 & 15.12 & 21.75 & 23.88 & 9.38 & 3.19 \\
\hline & $\begin{array}{l}\text { Overall Average Absolute De- } \\
\text { viation }(O A A D)\end{array}$ & & 29.04 & 58.51 & 34.50 & 43.96 & 31.66 & 26.13 & 38.38 & $\underline{5.10}$ \\
\hline & $\begin{array}{l}\text { Mean Percentage Deviation } \\
(M P D)\end{array}$ & & -6.93 & 52.65 & 16.24 & 32.20 & 15.07 & -0.05 & 30.22 & 0.64 \\
\hline & Number of points & 1079 & & & & & & & & \\
\hline
\end{tabular}


Table S5: Number of fluids in Table S4 with an AADs less than the value given in the first column. Sastri and Rao (SR) Eq. (2), ${ }^{21}$ Miqueu et al (MQ) Eq. (3), ${ }^{22}$ Di Nicola and Moglie (DM1,DM2) Eqs. (4) and (6), ${ }^{23}$ Gharagheizi al (GH) Eq. (7), ${ }^{24}$ Di Nicola et al (DCP1,DCP2) Eq. (8), ${ }^{15}$ and this work Eq. (24).

\begin{tabular}{l|r|r|r|r|r|r|r|r|} 
& SR & MQ & DM1 & DM2 & GH & DCP1 & DCP2 & Eq. (24) \\
\hline$<1.0 \%$ & 0 & 1 & 0 & 0 & 0 & 1 & 0 & 6 \\
\hline$<2.0 \%$ & 0 & 3 & 0 & 2 & 2 & 3 & 0 & 14 \\
\hline$<3.0 \%$ & 0 & 3 & 2 & 3 & 6 & 4 & 1 & 22 \\
\hline$<5.0 \%$ & 5 & 6 & 4 & 3 & 14 & 7 & 2 & 42 \\
\hline$<7.5 \%$ & 5 & 8 & 12 & 7 & 17 & 13 & 4 & 59 \\
\hline$<10.0 \%$ & 12 & 9 & 17 & 10 & 23 & 22 & 12 & 64 \\
\hline$<15.0 \%$ & 25 & 12 & 30 & 16 & 34 & 33 & 25 & 68 \\
\hline$<20.0 \%$ & 36 & 15 & 36 & 29 & 39 & 39 & 36 & 68 \\
\hline$\geq 20.0 \%$ & 33 & 55 & 34 & 41 & 30 & 31 & 34 & 2 \\
\hline \hline TOTAL & 69 & 70 & 70 & 70 & 69 & 70 & 70 & 70 \\
\hline
\end{tabular}



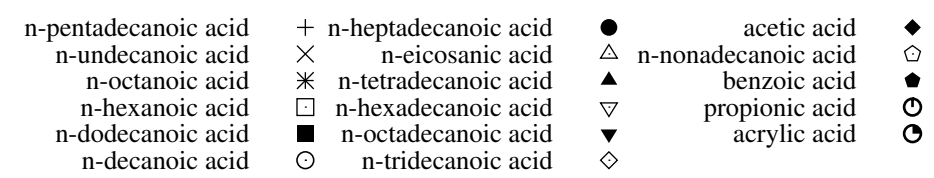

SR
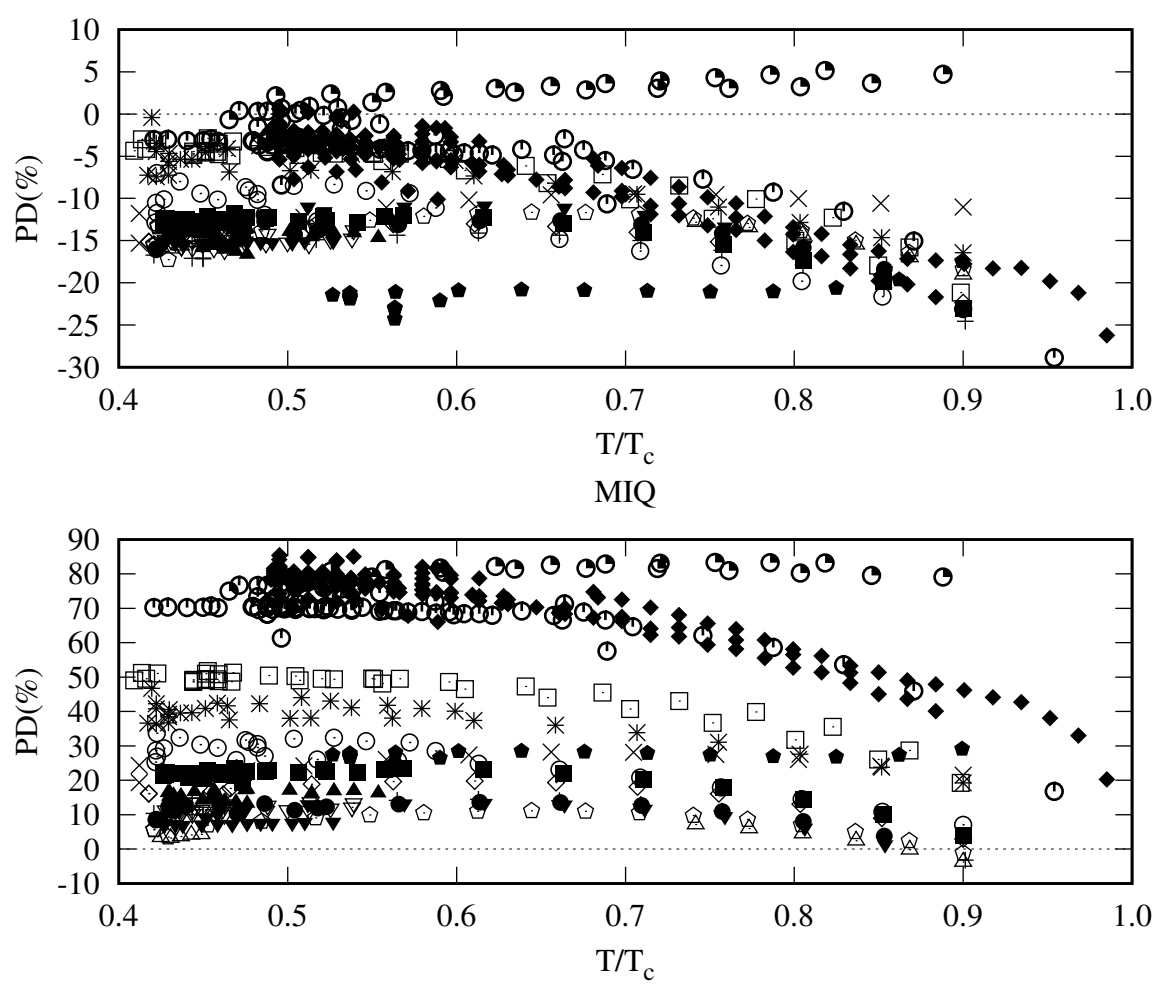

DM1

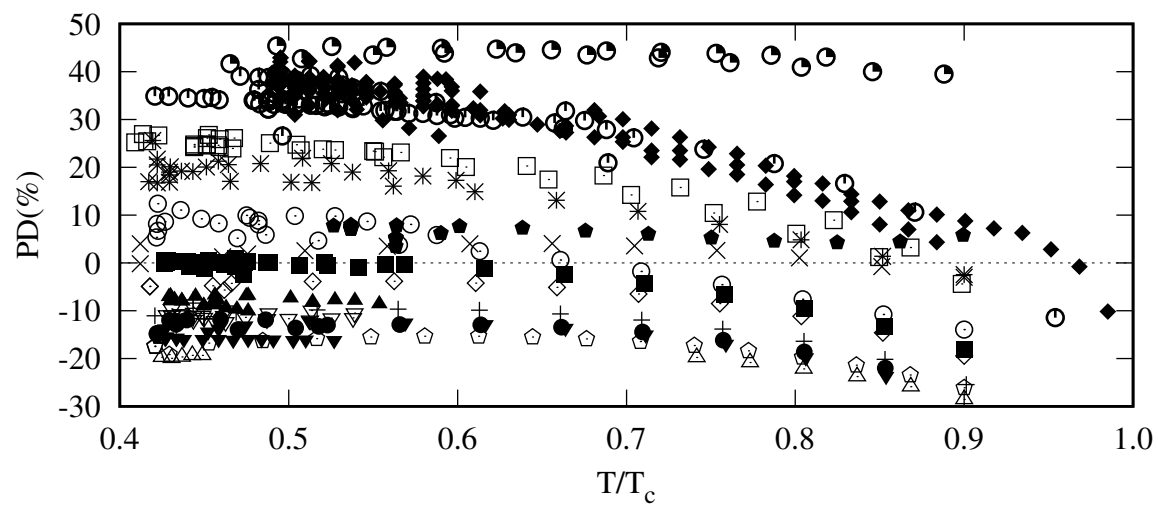

Figure S1: Percentage deviation of models SR, MIQ, DM1, respect the data of the 17 fluids in Table 1. 

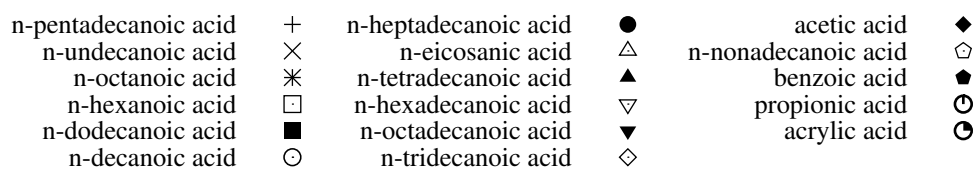

DM2
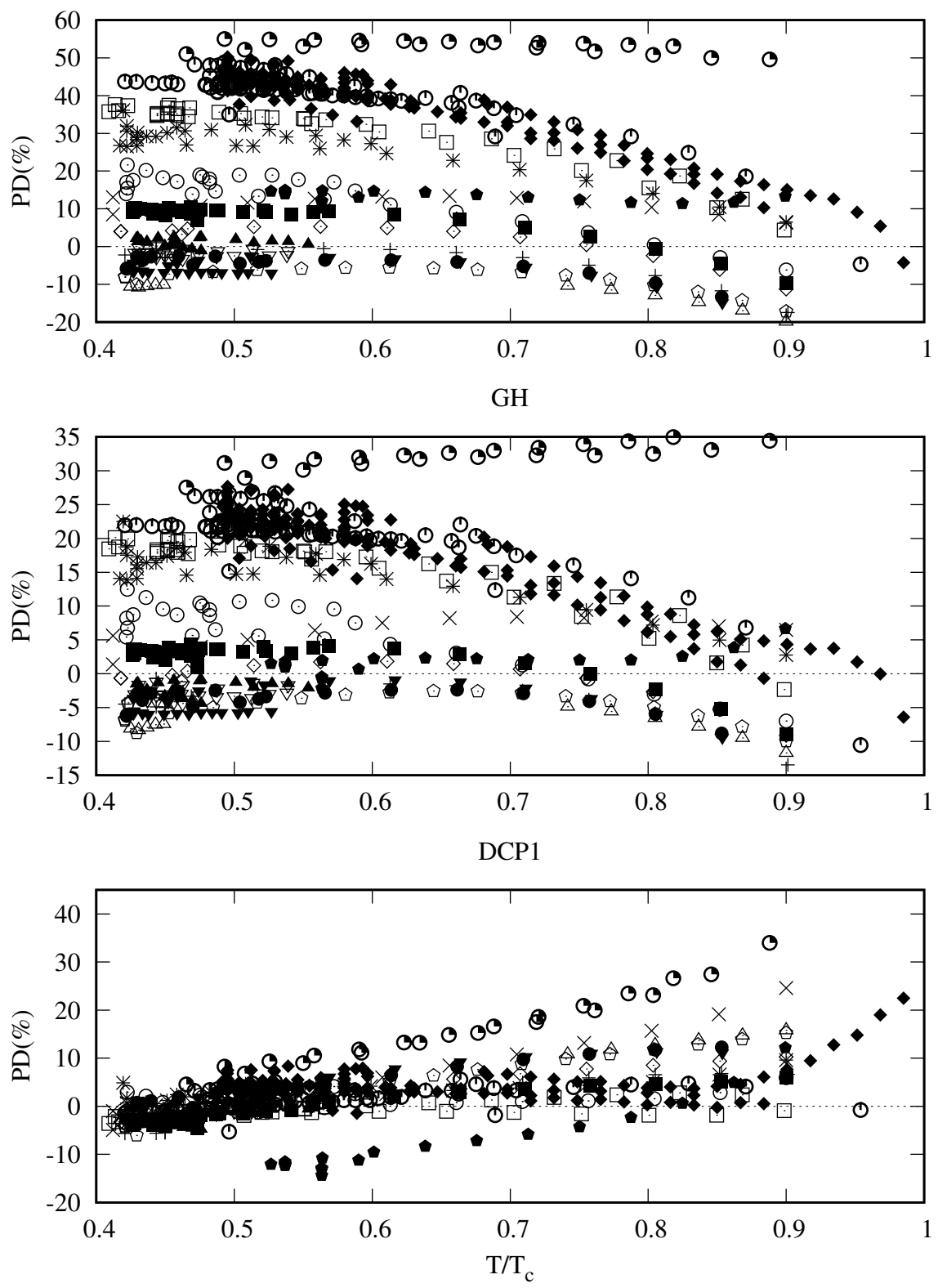

Figure S2: Percentage deviation of models DM2, GH and DCP1 of the 17 fluids considered in Table 1. 

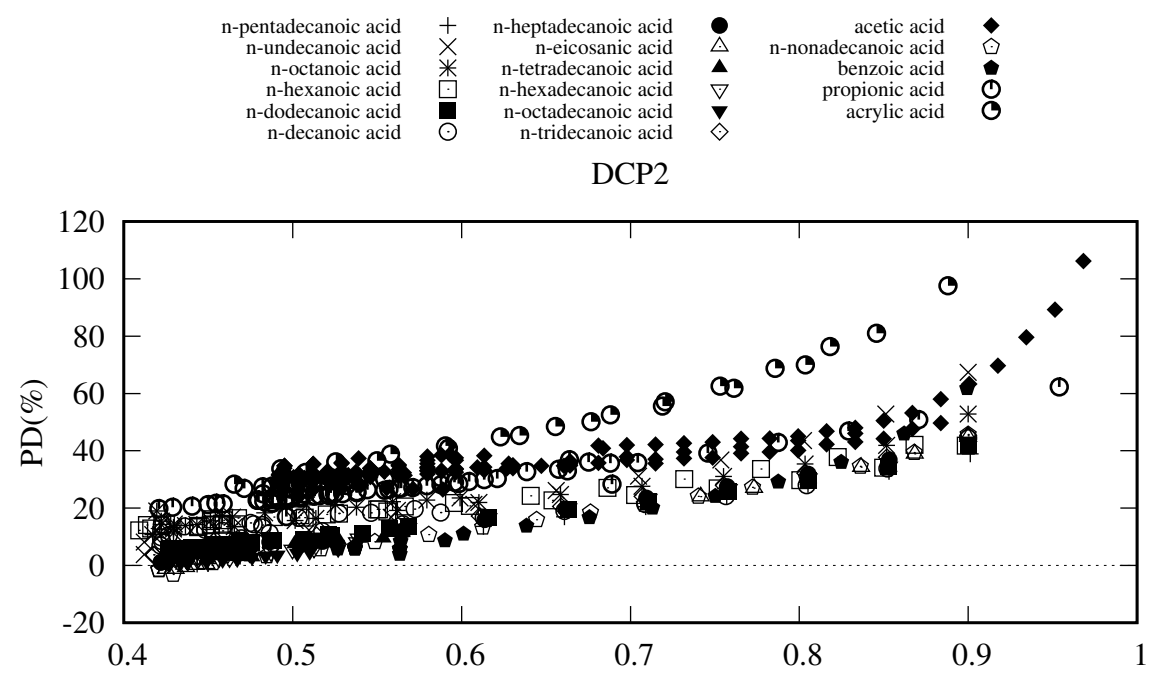

This work

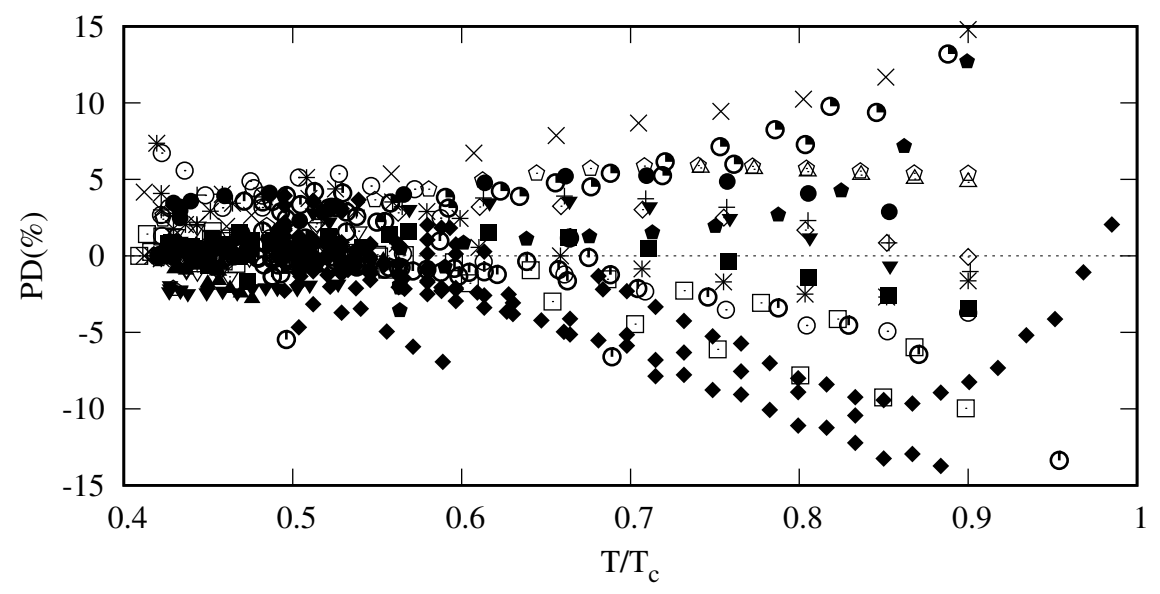

Figure S3: Percentage deviation of models DCP2 and this work eq. (24), respect the data of the 17 fluids in Table 1. 

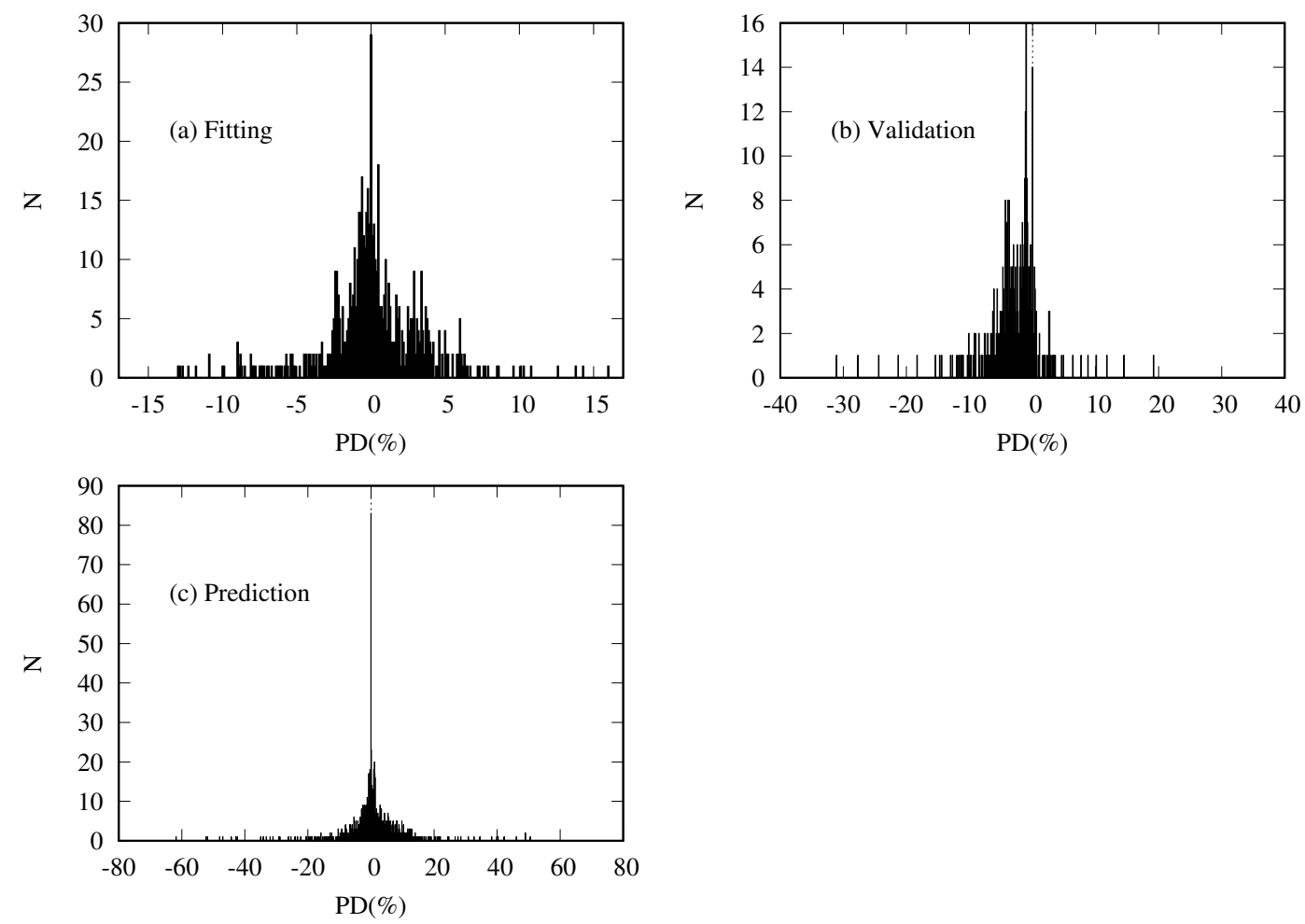

Figure S4: Histogram of the number of predicted data with a percentage deviation (PD) taken at $0.1 \%$ steps. (a) Fitting set, (b) Validation set, (c) Prediction set.

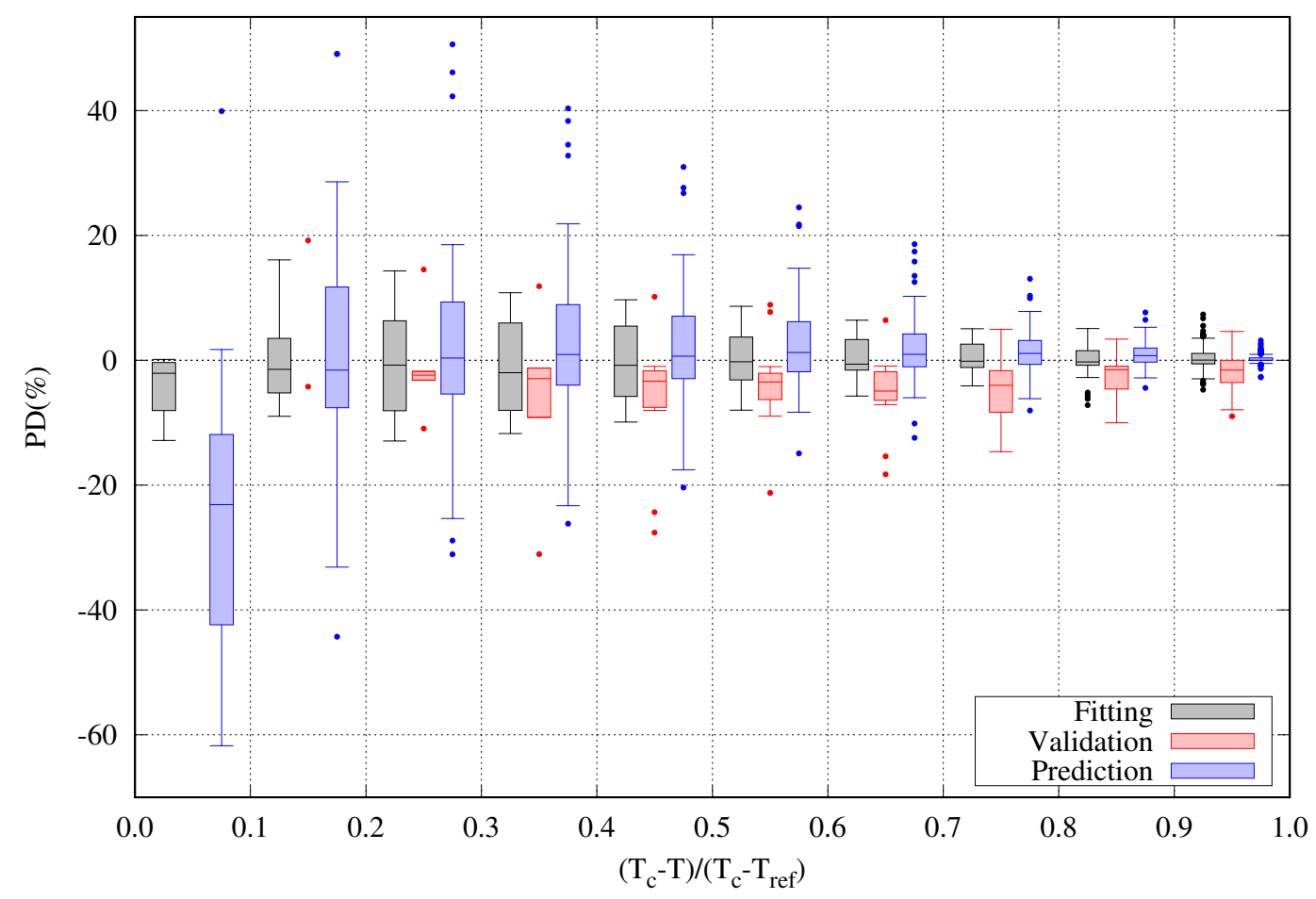

Figure S5: Box and whisker plot of the three sets considered taken at $0.1 T^{* *}$ steps. 

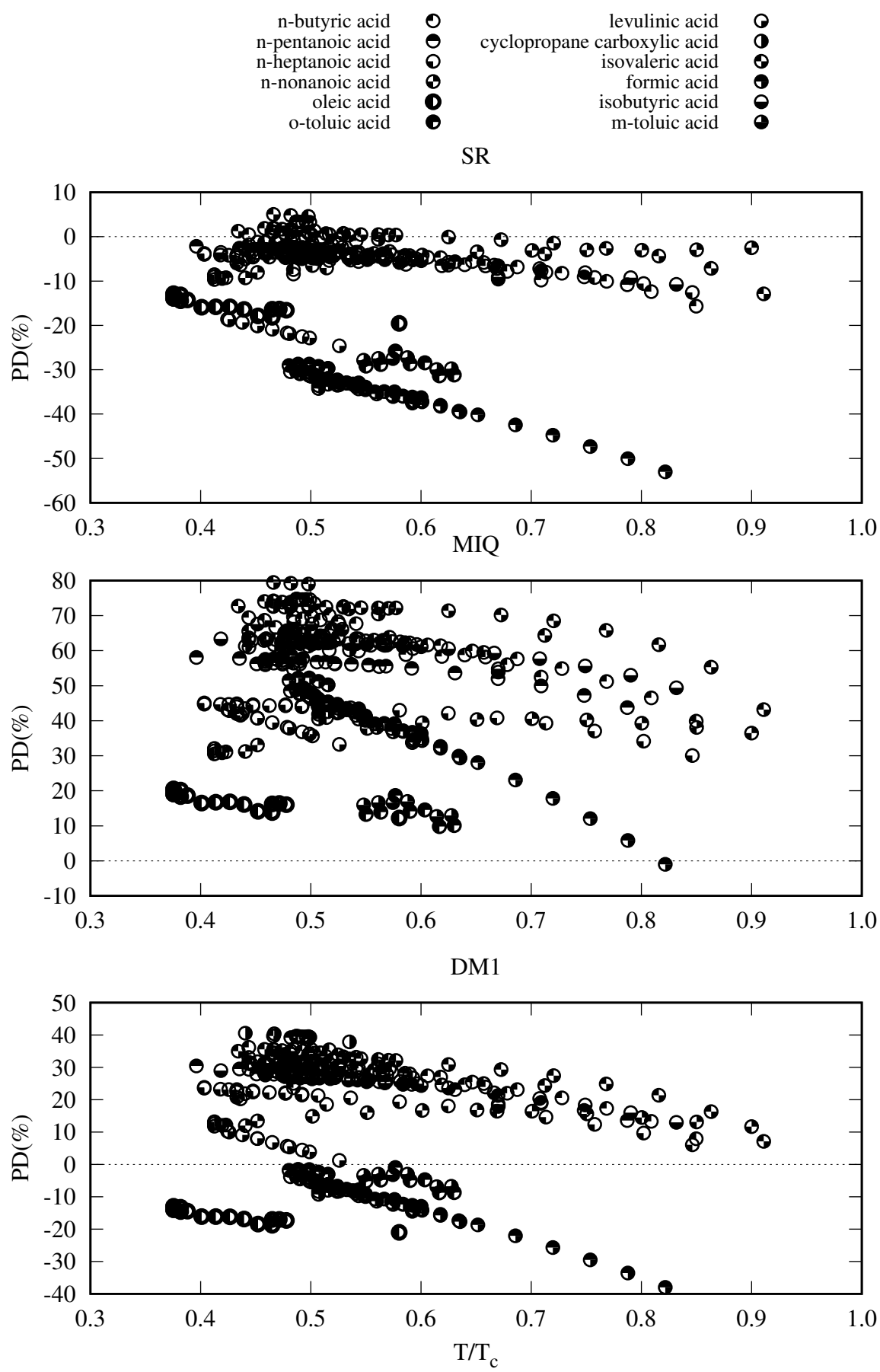

Figure S6: Percentage deviation of models SR, MIQ, DM1, respect the data of the 12 fluids in Table 2. 

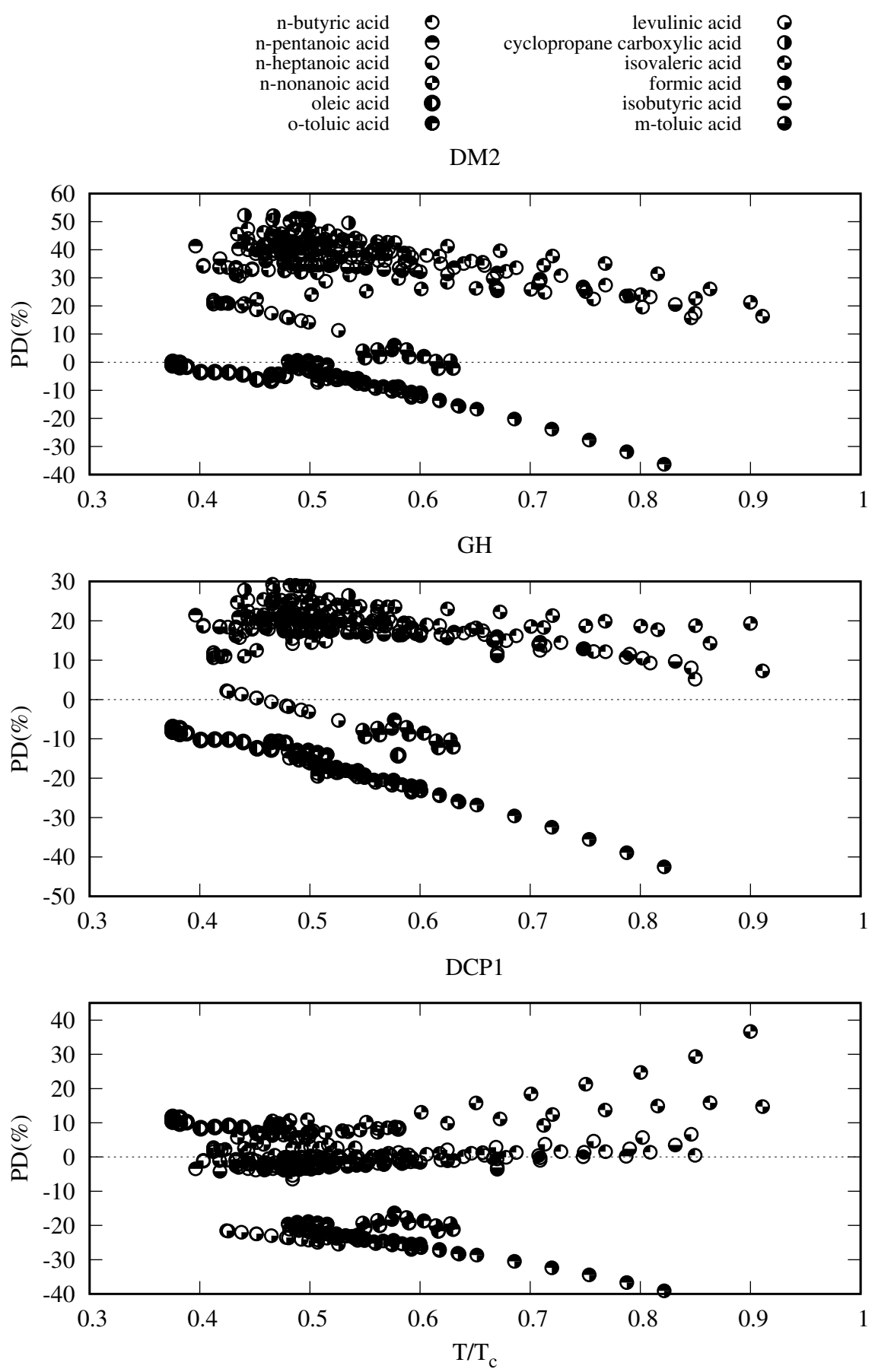

Figure S7: Percentage deviation of models DM2, GH and DCP1 of the 12 fluids considered in Table 2. The corresponding data of cyclopropane carboxilic acid, with percentage deviations about 400\%, are not shown in the DCP1 graph. 

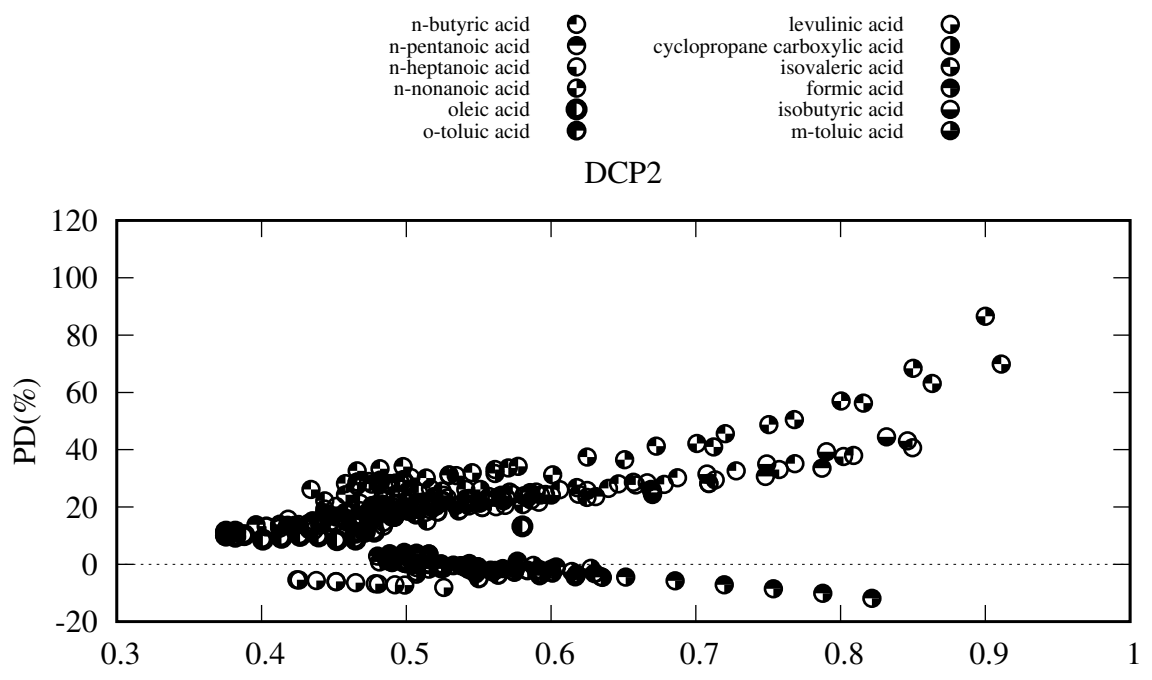

This work

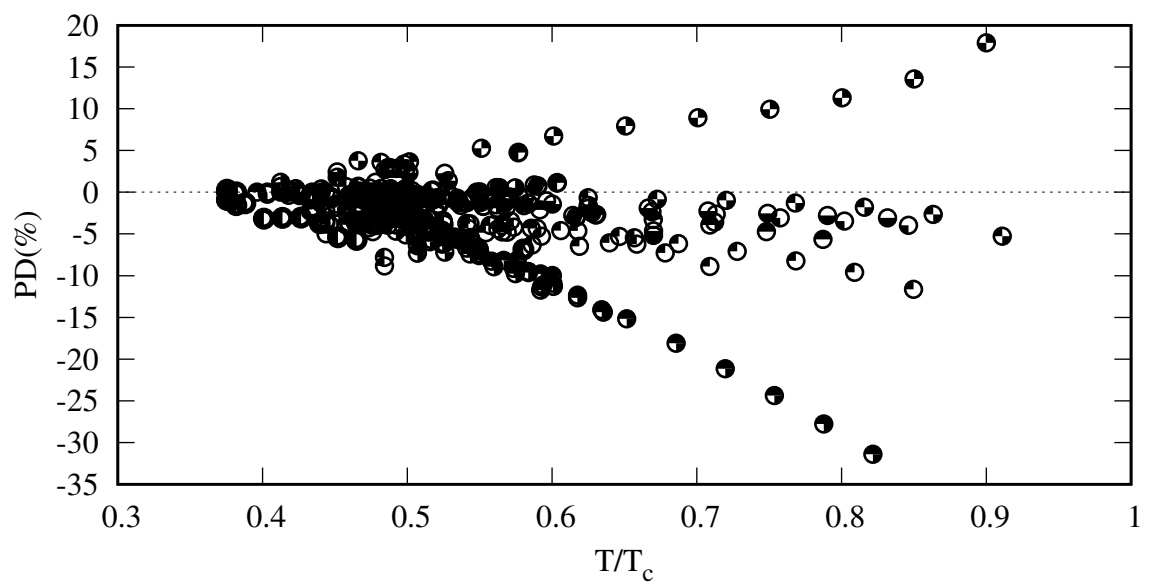

Figure S8: Percentage deviation of models DCP2 and this work eq. (24) of the 12 fluids considered in Table 2. The corresponding data of cyclopropane carboxilic acid, with percentage deviations about 300\%, are not shown in the DCP2 graph. 

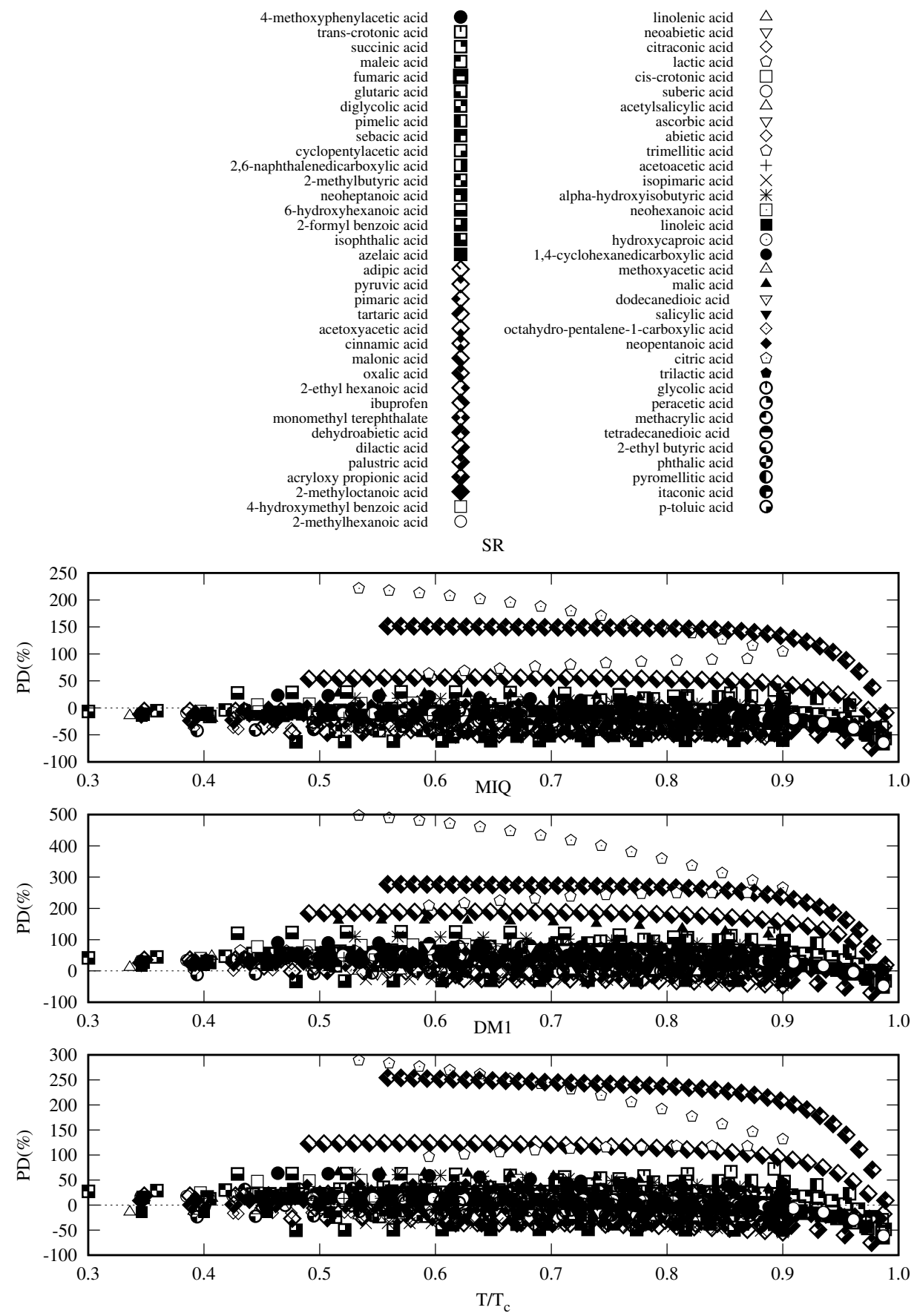

Figure S9: Percentage deviations of models SR, MIQ and DM1 with respect to the data of the 70 fluids in Table S3. 

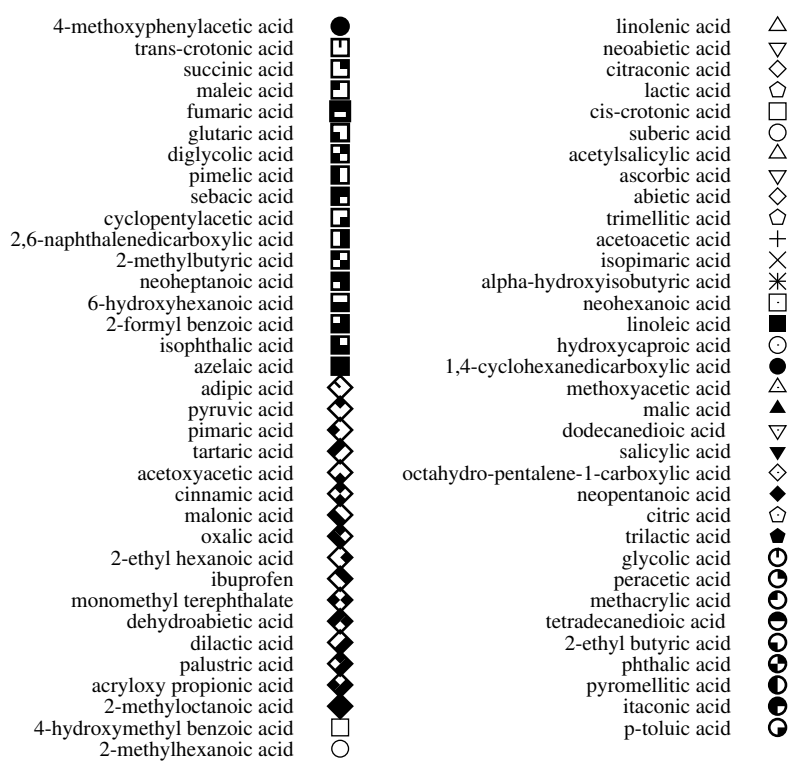

DM2
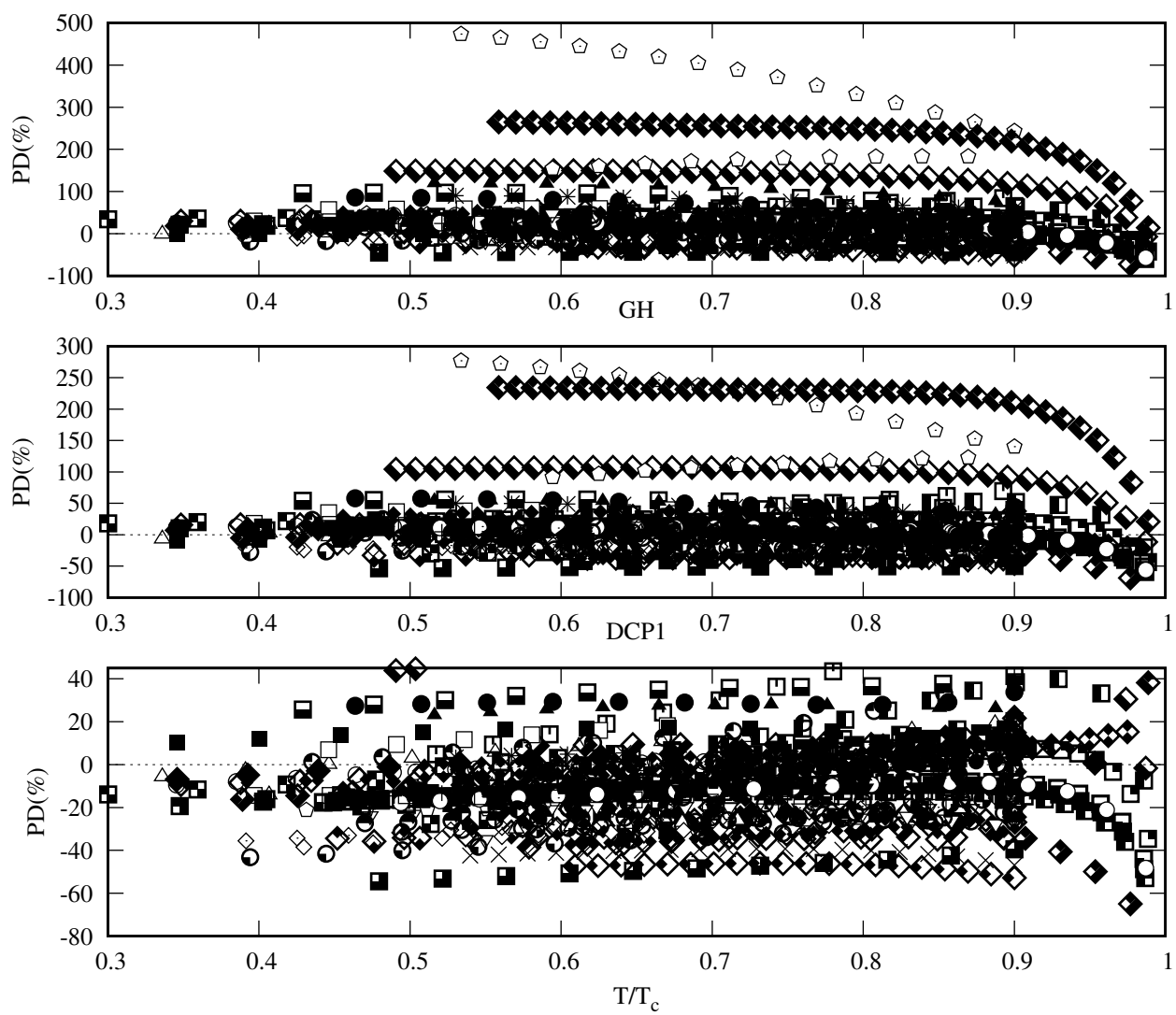

Figure S10: Percentage deviations of models DM2, GH, and DCP1, with respect to the data of the 70 fluids in Table S3. 

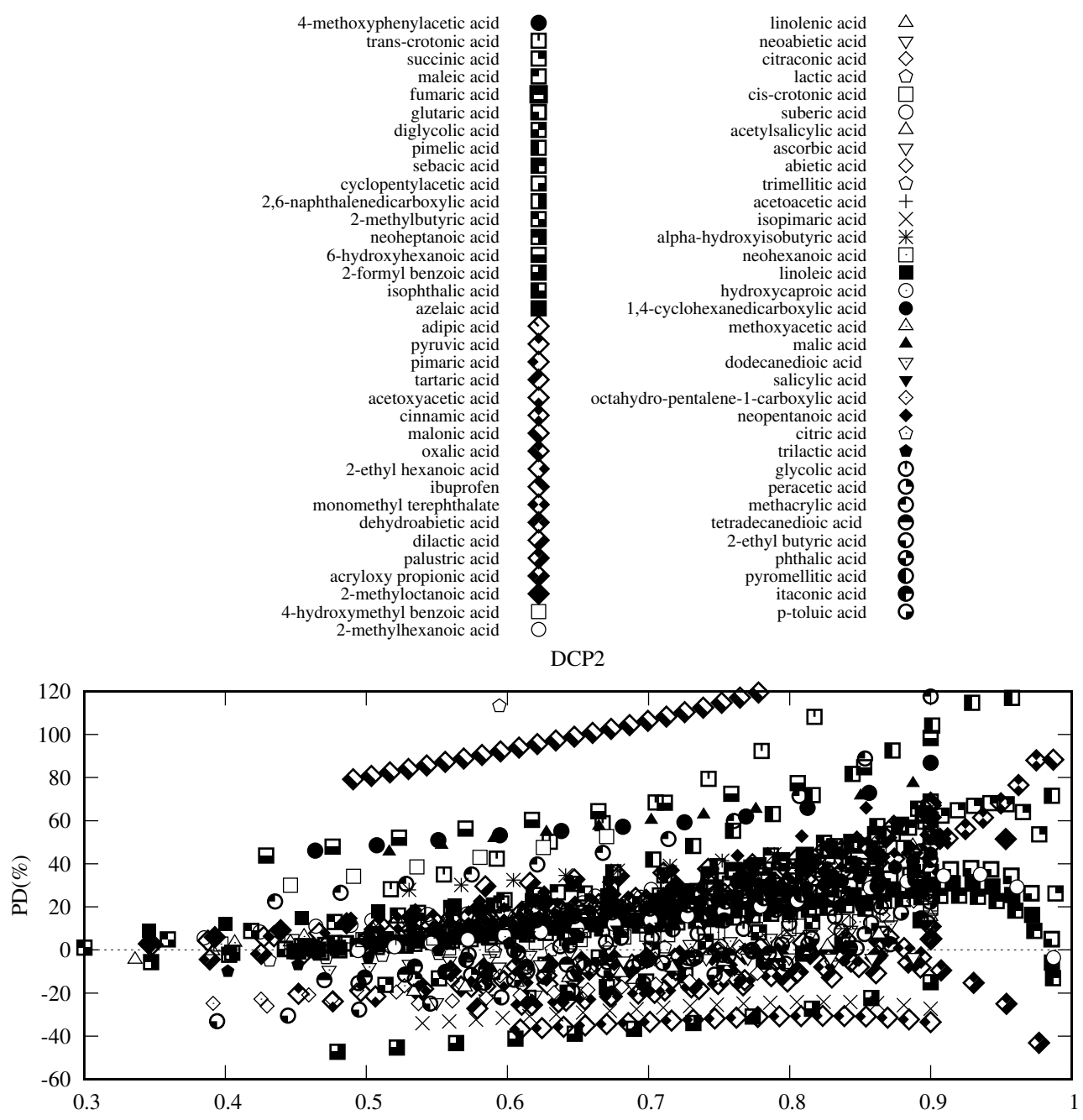

This work

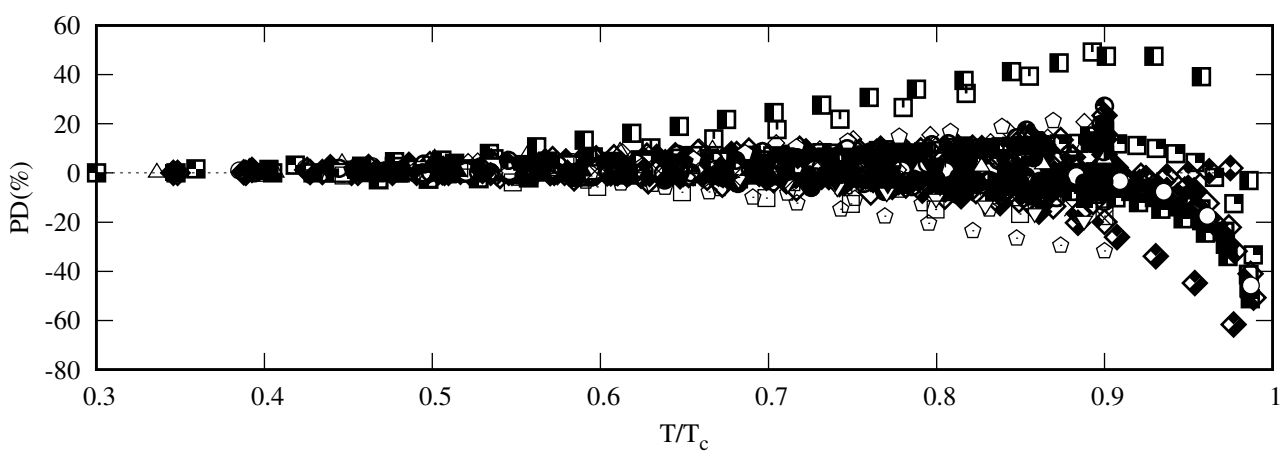

Figure S11: Percentage deviations of models DCP2 and this work Eq. (24) with respect to the data of the 70 fluids in Table S3 